\title{
High Altitude Remains Associated with Elevated Suicide Rates after Adjusting for Socioeconomic Status: A Study from South Korea
}

\author{
Jaelim Kim ${ }^{1,2 *}$, Nari Choi ${ }^{3 *}$, Yu-Jin Lee ${ }^{4}$, Hyonggin $\mathrm{An}^{3}$, \\ Namkug Kim ${ }^{5}$, Ho-Kyoung Yoon ${ }^{1}$, and Heon-Jeong Lee ${ }^{1 凶}$ \\ 'Department of Psychiatry, Korea University College of Medicine,Seoul, Republic of Korea \\ ${ }^{2}$ Department of Medicine, Korea University School of Medicine, Seoul, Republic of Korea \\ ${ }^{3}$ Department of Biostatistics, Korea University College of Medicine, Seoul, Republic of Korea \\ ${ }^{4}$ Department of Psychiatry, Seoul National University College of Medicine,Seoul, Republic of Korea \\ ${ }^{5}$ Department of Radiology and Research Institute of Radiology, University of Ulsan College of Medicine, Asan Medical Center, Seoul, Republic of Korea
}

There have been several studies supporting a possible relationship between high suicide rate and high altitude. However socioeconomic status may confound this association because low socioeconomic status, which is known to be related to a high suicide rate, is also associated with living at high altitude. This study aims to explore whether the relationship between high altitude and high suicide rate remains after adjusting for socioeconomic status in South Korea. We collected demographic data of completed suicides, the mean altitude of the district where each suicide took place, and the mean income of each district. We analyzed the data using regression analysis before and after adjustment for mean income. We found that there is a positive correlation between altitude and suicide rate, even after adjustment for mean income. Thus, altitude appears to be an independent risk factor for suicide.

Psychiatry Investig 2014;11(4):492-494

Key Words Suicide rate, Altitude, Socioeconomic status, South Korea.

\section{INTRODUCTION}

There have been several studies that support a possible positive relationship between high suicide rate and high altitude. ${ }^{1,2}$ Kim et al. ${ }^{1}$ reported that altitude appears to be a significant independent risk factor for suicide in the US when several predictor variables such as altitude, gun ownership, and population density are considered. They reported that both firearm and non-firearm suicide rates are positively correlated with mean county elevation. Brenner et al. ${ }^{2}$ also concluded that higher altitude was still significantly related to higher suicide rates, even after controlling for age, gender, ethnicity, median household income, and the population density of each county. Regarding the mechanism underlying this association, researchers have suggested that the effects of metabolic stress

Received: November 12, 2013 Revised: December 9, 2012

Accepted: December 9, 2013 Available online: October 20, 2014

$\triangle$ Correspondence: Heon-Jeong Lee, $\mathrm{MD}, \mathrm{PhD}$

Department of Psychiatry, Korea University College of Medicine, 73 Inchonro, Seongbuk-gu, Seoul 136-705, Republic of Korea

Tel: +82-2-920-6721, Fax: +82-2-929-7679, E-mail: leehjeong@korea.ac.kr

*The first two authors contributed equally to this study.

(c) This is an Open Access article distributed under the terms of the Creative Commons Attribution Non-Commercial License (http://creativecommons.org/licenses/bync/3.0) which permits unrestricted non-commercial use, distribution, and reproduction in any medium, provided the original work is properly cited. associated with mild hypoxia in individuals with mood disorders may be related to suicide. ${ }^{1}$

On the other hand, some researchers have suggested that the high suicide rates in high altitude areas are more likely to be the result of other confounding factors. Betz et al. ${ }^{3}$ found that high and low altitude suicide decedents differed with respect to race, ethnicity, rural residence, intoxication, firearm use, and recent financial, job, legal, or interpersonal problems. Therefore they suggested that other sociodemographic and mental health factors in high altitude communities, such as the relative availability and utilization of mental health resources, should be considered. ${ }^{3}$ Additionally, a study conducted in Turkey did not find any association between altitude and suicide. ${ }^{4}$ The author suggested that the positive results of previous studies may be due to confounding variables such as socioeconomic status. ${ }^{4}$

Based on the studies described above, an examination of how socioeconomic status contributes to high suicide rates in high altitude areas seems warranted. Furthermore, Kim et al. ${ }^{1}$ also reported the correlation between altitude and suicide rates in South Korea for a 4-year period (2005-2008) in their complementary studies.

The aim of this study was to determine the association be- 


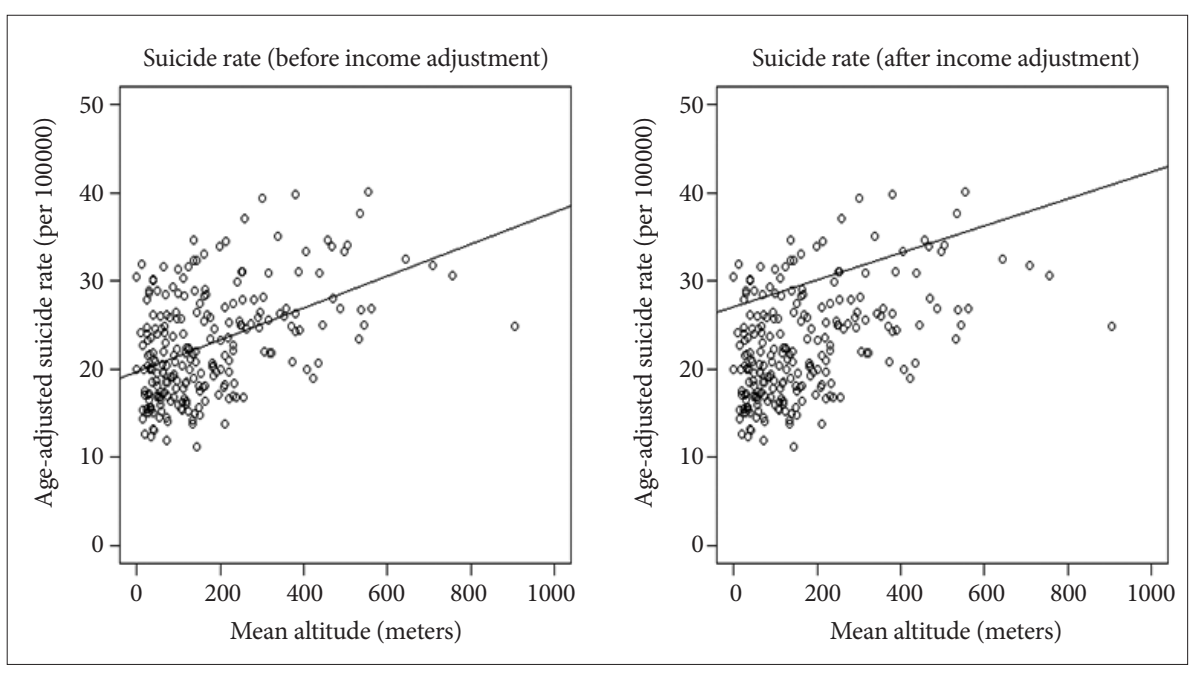

Figure 1. Scatter plot and regression line of mean altitude and age-adjusted suicide rate in 1997-2007 before and after income adjustment. tween high altitude and high suicide rates in South Korea after adjusting for socioeconomic factors using 11 years (19972007) of past data. The high suicide rates ${ }^{5}$ and mountainous geographic features with a wide range of mean altitudes among different districts make South Korea a suitable country in which to investigate whether high altitude is a significant independent risk factor for suicide.

\section{METHODS}

\section{Data collection}

Suicide rates and demographic information on suicide decedents, including sex, age, address, and date of death from 1997 to 2007, were collected from the National Statistical Office of South Korea. ${ }^{5}$ In this study, suicide rate was defined as the number of completed suicides per a hundred thousand persons in a given year. The mean altitudes of 231 administrative districts were collected from the National Geographic Information Institute. ${ }^{6}$ The data regarding the income of each district was collected from the Korean labor and income panel study. ${ }^{7}$ In this study, mean income implies the mean of each year's total income from 1998 to 2008 . In cases of missing income data, the mean income of adjacent administrative divisions was substituted. For example, when income data of a particular city was missing, the mean income of other cities in the same province was used.

\section{Statistical analyses}

The data was analyzed using SAS 9.3 (Cary, NC, USA) and R 2.15.3. First, we determined the Pearson's and Spearman's correlation coefficients between altitude and suicide rate. Afterward, regression analyses were performed to explore whether mean altitude affects age-adjusted suicide rate and income-and-age-adjusted suicide rate. In the first analysis, mean altitude was used as the independent variable and age-adjust- ed suicide rate was the dependent variable. Income was then added to the regression model as a covariate in an attempt to determine whether mean altitude is associated with suicide rate when financial factors are eliminated.

\section{RESULTS}

There was a significant positive correlation between ageadjusted suicide rate and the mean altitude of each district in South Korea [Pearson's correlation coefficient $=0.462(\mathrm{p}<$ 0.0001); Spearman's correlation coefficient $=0.419(\mathrm{p}<0.0001)]$. Regression analysis revealed that the age-adjusted suicide rate increased by $1.8 \%(\mathrm{p}<0.0001)$ for every one meter increase in mean altitude. When the mean income of each district was added as a covariate, income-age-adjusted suicide rate increased by $1.5 \%(\mathrm{p}<0.0001)$ for every one meter increase in mean altitude. The effect of mean altitude on suicide rate, which is represented by the slope of the graphs in Figure 1 , became a little less prominent as financial factors were eliminated. However, the change was minor, and the positive association remained significant after income adjustment.

\section{DISCUSSION}

The results of this study suggest that living at high altitude is a risk factor for suicide. This finding is consistent with the results of earlier studies. ${ }^{1,2}$ Previously, Kim et al. ${ }^{1}$ analyzed suicide data from a four-year period (2005-2008) in South Korea and reported a strong positive association between suicide rate and altitude in both South Korean and American populations. Our study also found a positive relationship between high altitude and suicide in South Korea based on data from a longer-period of time (1997-2007). On the other hand, Selek did not find an association between altitude and suicide in Turkey, and he suggested that the positive results of previous 
studies were probably due to confounding variables, such as differences in socioeconomic status. ${ }^{4}$ However, the positive association between mean altitude and suicide rate remained significant even after adjusting for socioeconomic factors in our study.

Our positive findings indicate that it would be meaningful to explore the mechanism underlying this association. Metabolic stress associated with hypoxia has been proposed as a possible mechanism. DelMastro et al. ${ }^{9}$ concluded that elevation is a significant risk factor for major depressive disorder, which is often associated with suicide. Similarly, other previous studies have described the impact of mean altitude on the metabolic status of individuals who have major mood disorders, especially bipolar disorders. The authors suggested that hypoxia due to high altitude aggravates affective disorders, ${ }^{2,8}$ which may lead to a higher likelihood of committing suicide.

It was suggested that serotonin synthesis is influenced by mild hypoxia. ${ }^{10}$ Tryptophan hydroxylase, which is the ratelimiting enzyme in the serotonin synthesis reaction, may not normally be saturated with oxygen at high altitudes, thus mild hypoxia would decrease serotonin synthesis. Low brain serotonin is known to be associated with suicide. Decreased brain serotonin synthesis associated with hypoxia is a mechanism that may contribute to suicide in conditions causing hypoxia. ${ }^{10}$ Fiedler et al. ${ }^{11}$ demonstrated that a significant correlation between altitude of region of residence and incidence of cocaine use. According to the authors, the stress response due to hypoxia is responsible for it and the positive correlation between altitude and incidence of cocaine use may partially explain how high altitude is linked to increased suicide risk.

Efforts to discover the mechanism of the association between altitude and suicide rate would be useful in suicide prevention among people living at high altitudes. However, this mechanism remains to be elucidated. Further studies to address this issue are necessary.

\section{Acknowledgments}

This research was supported by a grant of the Korea Health Technology R\&D Project through the Korea Health Industry Development Institute (KHIDI), funded by the Ministry of Health \& Welfare, Republic of Korea (HI12C1838) and partially supported by the intramural fund from Korea University.

\section{REFERENCES}

1. Kim N, Michelson JB, Brenner BE, Haws CA, Yurgelun-Todd DA, Renshaw PF. Altitude, gun ownership, rural areas, and suicide. Am J Psychiatry 2011;168:49-54

2. Brenner B, Cheng D, Clark S, Camargo CA Jr. Positive association between altitudes and suicide in 2568 U.S. counties. High Alt Med Biol 2011;12:31-35.

3. Betz ME, Valley MA, Lowenstein SR, Hedegaard H, Thomas D, Stallones L, et al. Elevated suicide rates at high altitude: sociodemographic and health issues may be to blame. Suicide Life Threat Behav 2011;41: 562-573.

4. Selek S. Altitude, immigration and suicude rates: a study from Turkey. Psychiatry Investig 2013:10:89-91.

5. Causes of Death by City, County, Gu-Based Statistics, South Korea (online database), Statistics Korea, 1997-2007. Available at: http://kostat. go.kr.

6. City, County, Gu Boundary Files (online database). Korean National GIS, National Geographic Information Institute, Ministry of Land, Transport, and Maritime Affairs, South Korea, 2010. Available at: http:// www.ngis.go.kr.

7. Korea Labor Institute. Korean Labor and Income Panel Study. Available at: http://www.kli.re.kr/.

8. Haws CA, Gray DD, Yurgelun-Todd DA, Moskos M, Meyer LJ, Renshaw $\mathrm{PF}$. The possible effect of altitude on regional variation in suicide rates. Med Hypotheses 2009;73:587-590.

9. DelMastro K, Hellem T, Kim N, Kondo D, Sung YH, Renshaw PF. Incidence of major depressive episode correlates with elevation of substate region of residence. J Affect Disord 2011;129:376-379.

10. Young SN. Elevated incidence of suicide in people living at altitude, smokers and patients with chronic obstructive pulmonary disease and asthma: possible role of hypoxia causing decreased serotonin synthesis. J Psychiatry Neurosci 2013;38:423-426.

11. Fiedler KK, BS, Kim N, Kondo DG, Renshaw PF. Cocaine use in the past year is associated with altitude of residence. J Addict Med 2012; 6:166-171. 